\title{
Automated Sorting Technology from T4T Can Help Improve Recovery and Efficiency
}

\section{Alkazam*}

Assistant researcher, Fabrics Laboratory, University of the Virgin Islands, USA

\begin{abstract}
Textile industry is one of the fastest growing sectors with a turnover of over $\$ 1$ trillion globally. The boom in this industry is seen very recently unlike other industries. This industry marked its infancy some 20 decades ago when old worn out clothes were modified to be used for different purposes. Textile industry usually consists of fabrics and clothing that highlights the durability and recycling characteristics of the material. Several different types of materials are being merged to form new material that have better durability and resistivity against physical parameters like heat, cold and other features.
\end{abstract}

Keywords: Textiles; Clothes; Fabrics; Durability; Recovery; Efficiency; Dyes

\section{Introduction}

The basis for the growing textile recycling industry is of course the textile industry itself. The textile industry has evolved into a $\$ 1$ trillion industry globally, comprising clothing, as well as furniture and mattress material, linens, draperies, cleaning materials, leisure equipment and many other items. Unlike mature industries such as metal recycling that have a long history, the growth of textile recycling is much more recent. Textile recycling was practiced some 200 years ago in England by "rag and bone men" who collected old clothing to be used in a number of ways.

\section{The urgency to recycle textiles}

The importance of recycling textiles is increasingly being recognized. In Canada, for example, over $\$ 30$ billion is spent on new clothing each year, translating to approximately 1.13 billion garments, and with an average growth rate of 5.16 percent per year. On average, Canadians discard seven kilograms (over 15 lbs.) of clothing per capita each year. Textiles constitute five percent of municipal solid waste by weight. As such, they, along with other segments of the solid waste stream such as organics are a significant segment to be addressed as we strive to move closer to a zero landfill society.

Natural fibers can take hundreds of years to decompose, and once in the landfill may release methane and $\mathrm{CO}_{2}$ gas into the atmosphere. The even more durable textiles manufactured from synthetics are designed not to decompose. In the landfill they may release toxic substances into groundwater and surrounding soil. During production, synthetics release nitrous oxide, which is a much more powerful greenhouse gas than $\mathrm{CO}_{2}$, absorbing as much as 270 times more heat [1].

For this reason, textile recycling offers the following environmental benefits:

- Decreases landfill space requirements, bearing in mind that synthetic fiber products do not decompose, and that natural fibers may release greenhouse gases

- Avoided use of virgin fibers

- Reduced consumption of energy and water

- Pollution avoidance

- Lessened demand for dyes.

\section{Sources of Textiles for Recycling}

Textiles for recycling are generated from two main sources.

These include:

- Post-consumer, including garments, vehicle upholstery, household items and others.

- Pre-consumer, including scrap created as a by-product from yarn and fabric manufacture, as well as the post-industrial scrap textiles from other industries.

The donation of old garments is supported by a number of nonprofit as well as corporate programs, such as the recycling programs of Nike and Patagonia.

After the collection of textiles takes place, an important step of the recycling process is the manual sortation of material, based on type of fiber and condition. Sorting also identifies wearable textiles such as usable shoes and clothes, as well as unwearable textiles that are destined for shredding and re-spinning.

\section{Which Recycling are Done in Textile}
1. Used Clothing
2. Used Footwear
3. Leather Recycling
4. Textile Recycling Employment
5. Other Textile Waste
6. Cotton Recycling
7. Wool Recycling

*Corresponding author: Alkazam, Assistant researcher, Fabrics Laboratory, University of the Virgin Islands, USA, E-mail: alkazama2@lab.viu.ac.us

Received June 27, 2013; Accepted September 03, 2013; Published September 09, 2013

Citation: Alkazam (2013) Automated Sorting Technology from T4T Can Help Improve Recovery and Efficiency. J Textile Sci Eng 3: 136. doi:10.4172/21658064.1000136

Copyright: (C) 2013 Alkazam. This is an open-access article distributed under the terms of the Creative Commons Attribution License, which permits unrestricted use, distribution, and reproduction in any medium, provided the original author and source are credited. 

8. Burlap, Jute and Sisal Recycling
9. Polyurethane Foam Recycling
10. Polyester and Polyester Fiber Recycling
11. Nylon and Nylon Fiber Recycling
12. Other Synthetic Fiber Recycling
13. Carpet Recycling
14. Rags and Wipers
15. Used and Recycled Bags

\section{Wearable and Reused Textiles}

According to a U.K. industry source, about 50 percent of collected textiles are reused, and about 50 percent are recycled. About 61 percent of recovered wearable clothes are exported to other countries. In some African countries, as many as 80 percent of people wear used clothing. The issue of sending used clothing to Africa has generated some degree of controversy as to the benefits of such initiatives, where it can have a negative impact on local textile industries, native dress, and local waste generation [2].

In Canada, an estimated 10 percent of charitable contributions are sold by thrift stores, with another 90 percent of donated fabrics going to textile recyclers. Approximately 35 percent of donated clothing is made into industrial rags. The downside of such a use is after usage, they may well end up back in the waste stream [3].

\section{Recycling Process for Natural Fibers}

For textiles to be recycled there are basic differences for natural and synthetic fibers. For natural textiles:

- Incoming unwearable material is sorted by type of material and color. Color sorting results in fabric that does not need to be re-dyed. The colour sorting means no re-dying is needed, saving energy and avoiding pollutants.

- Textiles are then pulled into fibers or shredded, sometimes introducing other fibres into the yarn. Materials are shredded or pulled into fibres. Depending on the end use of the yarn, other fibres may be incorporated.

- The yarn is then cleaned and mixed through a carding process

- Then the yarn is re-spun and ready for subsequent use in weaving or knitting. Special Processes

- Some fibers are not spun into yards, however. Some are compressed for textile filling such as in mattresses.

In the case of polyester-based textiles, garments are shredded and then granulated, and processed into polyester chips. These are subsequently melted and and used to create new fibers for use in new polyester fabrics.

As society becomes more familiar with the hazards associated with sending old textiles to the landfill, and the demand and supply of recycled textiles continues to develop, it can be anticipated that the textile recycling industry will continue to grow.

\section{Textiles for Textiles Develops Garment Sorting Technology for Recycling}

The sorting process is a critical step in many types of recycling, from pallets to plastics and more. Better accuracy allows for greater value recovery through better separation of different grades of material. Automation of sorting additionally helps in reducing labor requirements.

The same issue around sortation holds true for the garment or textile recycling sector as well. Garment recycling is a key component of reducing the garment industry's carbon footprint, but its potential is still not fully realized due to a lack of technology being readily available to this application, particularly when it comes to sorting.

Garment recovery and reuse for charity purposes is an important component of the recycling effort, accounting for roughly 50 percent of recovery in countries such as the Netherlands, in addition to other clothing reuse niches. The other 50 percent, however, must be sortedstill often manually. In addition to the exhaustive labor requirements associated with manual sorting, difficulties in identifying specific fabrics have resulted in inadequately sorted textiles being used for generally lower grade applications such as stuffing or rags, rather than recovered for higher grade applications where such material could supplement the use of virgin fiber.

In order to address this need, Textiles for Textiles (T4T) has been developing an automated sorting technology for textiles and clothing, with support of European Commission's eco-innovation initiative, which helps fund a number of environmental initiatives, including materials recycling and recycling processes. Three years in development, the sorting system will be able to sort fabric material based on fiber composition as well as by color. This will be accomplished through the use of Near Infrared (NIR)-spectroscopy technology, which is widely used in automated sorting applications for other segments of the recycling industry, such as PET recycling. The system will cost in the order of 200,000 to 500,000 Euros (\$US 260,000 to $\$$ US 654,000 ) $[4,5]$.

After first going through a manual sorting process to segregate that clothing which is still usable and can be saved, the remaining garments are then are fed through the automatic sorting system for shredding. Resulting long fibres can be utilized in products such as clothing and home textiles, while shorter fibres are directed toward non-woven applications in insulation, personal hygiene and automotive.

The environmental impact reduction associated with the use of recycled fibers is impressive, according to the development group. In a pilot project with a jeans manufacturer, the energy savings of recycled fiber versus virgin was 53 percent, while the water saving amounted to 99 percent, and the chemical saving was 88 percent $[6,7]$.

\section{The Process of Recovering and Recycling Old Clothing}

Garment recycling is a key activity of textile recycling. It involves the process of recovering old clothing and shoes for sortation and recovery. End products include clothing suitable for reuse, processing into cloth scraps or rags as well as fibrous material. Interest in garment recycling is rapidly on the rise for a number of reasons, including environmental awareness, landfill pressure, as well as the economic opportunities presented to clothing recyclers as well as various charities that also generate revenue through their collection programs for old clothing [8].

Garment recycling involve a series of sequential activities. Following are the activities that garments recycling companies are to perform sequentially. 
Citation: Alkazam (2013) Automated Sorting Technology from T4T Can Help Improve Recovery and Efficiency. J Textile Sci Eng 3: 136. doi:10.4172/2165-8064.1000136

\section{Collection}

Clothing recyclers use a variety of strategies for picking up clothing. Post-consumer clothing is picked up generally from bins placed in public places, clothing drives, or door-to-door pickup. Bins are typically placed in public places like parking lots if business centers and shopping malls. Colorful bins are positioned in high traffic, high visibility locations to help maximize donations. Some charities also collect from homes, announcing prior when they will be picking up in a particular neighborhood, or utilizing volunteers to call residences on their list to see if there will be old clothing available for pickup.

One recent development has been the partnering of leading retailers with garment recycling companies such as typically these are placed in garbage bags and left at the front door of the residence for pickup. Other sources of clothing for recycling may include old industrial uniforms and linens, as well as pre-consumer scrap from garment manufacturing [9].

\section{Clothing sorting}

Once collected, clothing is classified into three kinds; reuse, rags, and fiber. Typically this is a manual sorting process that requires expertise in identifying various types of material. The process can be aided by such mechanical systems as conveyor belts and bins to segregate various grades of material. There is, however, at least one initiative to automate the sorting process, known as Textiles4Textiles. Recyclers report that about one-half of donated garments can be reused. Some recyclers bale this clothing for export to developing countries, while some garments are used domestically for sale in thrift shops. Industrial cloth rags and wipes are another important residual of the recycling process. Additionally, clothing may be reduced to fibrous material.

\section{Processing}

Textile fabric and clothing commonly consist of composites of synthetic plastics and cotton (biodegradable material). The composition will influence its method of recycling and durability.

Collected clothing are sorted and graded for reprocessing by highly experienced and skilled workers, who are capable of recognizing the huge selection of fiber types resultant from the beginning of blended and synthetics fiber fabrics. These sorted items are sent to different destinations as outlined.

Fiber renovation mills rate inward material into color and type. The shoring of colors means no re-dying is necessary which would save pollutants and energy. Then the clothing is torn into sloppy fibers and combined with other chosen fibers, conditional on the planned end use of the recycled fiber. The combined mixture is tagged to mix and clean the fibers and spun completely ready for knitting or weaving. These fibers can furthermore be compressed for production of mattress. Textiles which are sent to the flocking industry are used to produce filling material for furniture padding, panel linings, loudspeaker cones, and car insulation [10].

The recycling process works somewhat differently for specialized polyester based materials. In this case, the first thing is to remove zippers and buttons and then to cut the clothing into smaller pieces.
Those shredded small fabrics are then granulated and shaped into little pellets.

As the textile industry continues to grow, it will be challenged to devise ways to boost recycling rates as well as to develop technologies that will help maximize the value of recovered material.

\section{References}

1. Clifford YT, Chi-Kao W, Meng-Chun C (2008) Effects of magnetic field on the crystallization of $\mathrm{CaCO}_{3}$ using permanent magnets. Chemical Engineering Science 63: 5606-5612.

2. Dave PC (2004) Water-Industrial Water Purification, Technical Director "Water Softeners.

3. Duffy EA (1977) Investigation of Magnetic Water Treatment Devices. Ph.D Dissertation, Clemson University, Clemson, S.C.

4. Ghazi O, Julio TG (2010) An Overview of Magnetic Water Treatment System \& Further Course of Study. J Int Environmental Application \& Science 5: 965-974.

5. Arabshahi H (2011) The Effect of Magnetic Water on Strength Parameters of Concrete. An International Journal of Chemistry 6: 66-69.

6. Banejad H, Abdosalehi E (2009). The Effect of Magnetic Field on Water Hardness Reducing. Thirteenth International Water Technology Conference, IWTC 13, Hurghada, Egypt.

7. Hasson D, Bramson D (1985) Effectiveness of Magnetic Water Treatment in Suppressing $\mathrm{CaCO}_{3}$ Scale Deposition. Ind Eng Chem Process Des Dev 24 588-592.

8. Gholizadeha M, Arabshahib H, Benamc MR (2005) The Effect of Magnetic Field on Scale Prevention in the Industrial Boilers. International Journal of Applied Chemistry 1: 84-89.

9. Amiri MC, Ali AD (2006) On Reduction in the Surface Tension of Water Due to Magnetic Treatment. Colloids and Surfaces A: Physicochem. Eng. Aspects 278: $252-255$

10. Marshall SV, Skitek GG (1987) Electromagnetic Concepts and Applications (2nd edn.). Prentice-Hall, Englewood Cliffs, NJ, USA 